\title{
Electronic structure, magnetic ordering and X-ray magnetic circular dichroism in $\mathrm{La}_{1-x} \mathrm{Pr}_{x} \mathrm{Co}_{2} \mathrm{P}_{2}$ phosphides
}

\author{
L.V. Bekenov, S.V. Moklyak, V.N. Antonov \\ Institute of Metal Physics of the National Academy of Sciences of Ukraine, 36 Vernadsky Blvd., 03142 Kyiv, \\ Ukraine
}

Received January 31, 2015

The electronic structure and magnetic ordering in $\mathrm{La}_{1-x} \mathrm{Pr}_{x} \mathrm{Co}_{2} \mathrm{P}_{2}(x=0,0.25$, and 1$)$ phosphides have been studied theoretically using the fully relativistic spin-polarized Dirac linear muffin-tin orbital (LMTO) band-structure method. The X-ray absorption and X-ray magnetic circular dichroism spectra at the $\operatorname{Co} L_{2,3}$ and $\operatorname{Pr} M_{4,5}$ edges have been investigated theoretically within the framework of the LSDA $U$ method. The core-hole effect in the final state as well as the effects of the electric quadrupole $E_{2}$ and magnetic dipole $M_{1}$ transitions have been investigated. Good agreement with experimental measurements has been found.

Key words: strongly correlated systems, band structure, magnetic moments, X-ray magnetic circular dichroism

PACS: $71.28 .+d, 71.25 . P i, 75.30 . \mathrm{Mb}$

\section{Introduction}

Ternary intermetallics $\mathrm{AT}_{2} \mathrm{X}_{2}$, where $\mathrm{A}$ - alkali, alkali-earth, rare-earth, or actinide metal, $\mathrm{T}$ - transition metal, and $\mathrm{X}$ - nonmetal, often demonstrate intrinsically complex magnetic structures and a wide variety of physical properties. They belong to the $\mathrm{ThCr}_{2} \mathrm{Si}_{2}$ structure type and can be conveniently represented as materials built by stacking covalently bonded transition metal-metalloid $\mathrm{T}_{2} \mathrm{X}_{2}$ layers, made of edge-sharing $\mathrm{TX}_{4}$ tetrahedra, with ionic A atoms. This body-centered tetragonal structure is rather simple with only one crystallographic site for each atomic species and only one variable positional parameter, the $z$ value of the $\mathrm{X}$ site. Due to the great variety of possible elements in this structure, over 1000 different compounds of this type are known already [1]. Some of these compounds exhibit fascinating physical properties such as superconductivity, valence fluctuations, local and itinerant magnetism. Interest to these materials has been reinvigorated recently due to the discovery of non-Fermi-liquid behavior in $\mathrm{YbRh}_{2} \mathrm{Si}_{2}$ [2] and high-temperature superconductivity in K-doped $\mathrm{BaFe}_{2} \mathrm{As}_{2}$ [3, 4]. In addition, materials with layered arrangements of magnetic moments are also of great interest because they tend to exhibit a highly anisotropic magnetic behavior. The magnetic properties of the silicides and germanides possessing this structure were studied by many groups [5-11]. Besides superconductivity, $\mathrm{AT}_{2} \mathrm{X}_{2}$ phases exhibit a plethora of other phase transition phenomena. Thus, $\mathrm{SmMn}_{2} \mathrm{Ge}_{2}$ becomes ferromagnetic below $348 \mathrm{~K}$ and then undergoes a transition to an antiferromagnetic state at $196 \mathrm{~K}$, followed by a re-entrant ferromagnetic transition at $64 \mathrm{~K} \mathrm{[7].} \mathrm{This} \mathrm{peculiar} \mathrm{magnetic} \mathrm{behavior} \mathrm{stems} \mathrm{from} \mathrm{the} \mathrm{layered} \mathrm{structure}$ and the presence of two magnetic sublattices in these materials and was later observed for a number of rare-earth manganese germanides and silicides [8-11]. Such sequential magnetic transitions have not been observed in isostructural phosphides which might explain why their magnetic properties have received somewhat less attention than the tetrelides. The isotypic phosphides were prepared much later [12] and the investigations of their magnetic properties have started only recently.

In the compounds $\mathrm{RT}_{2} \mathrm{P}_{2}(\mathrm{R}=$ lanthanides, $\mathrm{T}=\mathrm{Fe}, \mathrm{Co}, \mathrm{Ni})$ with the trivalent rare earth elements, magnetic moments at the transition metal sites were observed only for the cobalt containing compounds 
[13-15]. Reehuis et al. carried out a comprehensive study of the structural and magnetic properties of ternary $\mathrm{RCo}_{2} \mathrm{P}_{2}$ materials [15-17]. Recently, Kovnir et al. [18-20] and other groups [21, 22] have demonstrated that the magnetic properties of the $\mathrm{ThCr}_{2} \mathrm{Si}_{2}$ type phosphides can be as rich and diverse as those of the aforementioned silicides and germanides, provided that proper iso- and aliovalent substitutions are made to modify the crystal and electronic structures of the materials. Ternary cobalt phosphides $\mathrm{RCo}_{2} \mathrm{P}_{2}$ appear to be on the verge of magnetic instability [20]. Indeed, $\mathrm{LaCo}_{2} \mathrm{P}_{2}$ is characterized by ferromagnetic ordering of Co magnetic moments at $132 \mathrm{~K}$ [14, 18], while other representatives of this family ( $\mathrm{R}=\mathrm{Ce}, \mathrm{Pr}, \mathrm{Nd}, \mathrm{Sm}$ ) order antiferromagnetically at around room temperature [15] (with the exception of the Ce-containing compound, which shows an antiferromagnetic transition at $440 \mathrm{~K}$ purportedly due to the mixed valence of (e). A substantial difference is also observed in the crystal structures of these compounds [23]. In $\mathrm{LaCO}_{2} \mathrm{P}_{2}$, the $\left[\mathrm{CO}_{2} \mathrm{P}_{2}\right]$ layers are far from each other, with the interlayer P-P distance of $3.16 \AA$ indicating essentially no bonding between the phosphorus atoms. By contrast, other $\mathrm{RCo}_{2} \mathrm{P}_{2}$ structures show a weakly covalent P-P interaction at relatively small interplanar P-P distances (2.57 $\AA$ in $\mathrm{PrCO}_{2} \mathrm{P}_{2}$ [18]). Thus, a drastic change in the magnetic behavior is accompanied by the formation of the $\mathrm{P}-\mathrm{P}$ bonding pathway between the $\left[\mathrm{Co}_{2} \mathrm{P}_{2}\right]$ layers.

Kovnir et al. [18] embarked on the study of structural, magnetic, and electronic properties of quaternary compositions $\mathrm{La}_{1-x} \mathrm{Pr}_{x} \mathrm{Co}_{2} \mathrm{P}_{2}(0 \leqslant x \leqslant 1)$. The choice of $\mathrm{Pr}$ was based on the fact that it does not exhibit a mixed valence like $\mathrm{Ce}$, and, therefore, is the closest rare-earth neighbor of La in both the size and the formal ionic charge. They focused on the drastically different magnetic properties of $\mathrm{LaCo}_{2} \mathrm{P}_{2}$ and $\mathrm{PrCO}_{2} \mathrm{P}_{2}$, which exhibit ferromagnetic $\left(T_{\mathrm{C}}=132 \mathrm{~K}\right)$ and antiferromagnetic $\left(T_{\mathrm{N}}=305 \mathrm{~K}\right)$ ordering in the Co sublattice, respectively [18]. In both cases, a ferromagnetic arrangement of magnetic moments within the square plane of $\mathrm{Co}$ atoms is observed. In $\mathrm{LaCO}_{2} \mathrm{P}_{2}$, the Co moments are aligned in-plane and parallel to the moments in the other layers, leading to the ferromagnetic ground state [17]. In $\mathrm{PrCo}_{2} \mathrm{P}_{2}$, they are aligned perpendicular to the plane (along the $c$ axis), but the antiparallel magnetic coupling between the neighboring planes results in antiferromagnetism [16]. In [24], magnetic behavior of $\mathrm{La}_{0.75} \mathrm{Pr}_{0.25} \mathrm{Co}_{2} \mathrm{P}_{2}$ was investigated by a combination of magnetic measurements, magneto-optical imaging, neutron diffraction, and X-ray absorption spectroscopy, including X-ray magnetic circular dichroism.

The aim of this paper is a theoretical study, from the first principles, of the electronic structure, magnetic ordering and X-ray magnetic circular dichroism in $\mathrm{La}_{1-x} \mathrm{Pr}_{x} \mathrm{Co}_{2} \mathrm{P}_{2}(x=0,0.25$, and 1$)$ compounds. The energy band structure of $\mathrm{La}_{1-x} \mathrm{Pr}_{x} \mathrm{Co}_{2} \mathrm{P}_{2}(x=0,0.25$, and 1$)$ compounds is calculated within the $a b$ initio approach taking into account strong electron correlations by applying the local spin-density approximation (LSDA) to the density functional theory supplemented by a Hubbard $U$ term $(\operatorname{LSDA}+U$ ) [25]. The paper is organized as follows. The computational details are presented in section 2 . Section 3 presents the electronic structure, XAS and XMCD spectra of $\mathrm{La}_{1-x} \mathrm{Pr}_{x} \mathrm{Co}_{2} \mathrm{P}_{2}(x=0,0.25$, and 1$)$ compounds calculated in the LSDA $+U$ approximation. The theoretical results are compared to experimental measurements. Finally, the results are summarized in section 4.

\section{Computational details}

X-ray magnetic circular dichroism. The absorption coefficient $\mu_{j}^{\lambda}(\omega)$ for incident X-ray of polarization $\lambda$ and photon energy $\hbar \omega$ can be determined as the probability of electronic transitions from initial core states with the total angular momentum $j$ to final unoccupied Bloch states:

$$
\mu_{\lambda}^{j}(\omega)=\sum_{m_{j}} \sum_{n \mathbf{k}}\left|\left\langle\Psi_{n \mathbf{k}}\left|\Pi_{\lambda}\right| \Psi_{j m_{j}}\right\rangle\right|^{2} \delta\left(E_{n \mathbf{k}}-E_{j m_{j}}-\hbar \omega\right) \theta\left(E_{n \mathbf{k}}-E_{\mathrm{F}}\right),
$$

where $\Psi_{j m_{j}}$ and $E_{j m_{j}}$ are the wave function and the energy of a core state with the projection of the total angular momentum $m_{j}$; $\Psi_{n \mathbf{k}}$ and $E_{n \mathbf{k}}$ are the wave function and the energy of a valence state in the $n$-th band with the wave vector $\mathbf{k} ; E_{\mathrm{F}}$ is the Fermi energy. $\Pi_{\lambda}$ is the electron-photon interaction operator in the dipole approximation

$$
\Pi_{\lambda}=-\mathrm{e} \boldsymbol{\alpha} \mathbf{a}_{\lambda},
$$

where $\boldsymbol{\alpha}$ are the Dirac matrices, $\mathbf{a}_{\lambda}$ is the $\lambda$ polarization unit vector of the photon vector potential, with $a_{ \pm}=1 / \sqrt{2}(1, \pm i, 0), a_{\|}=(0,0,1)$. Here, + and - denotes, respectively, left and right circular photon polarizations with respect to the magnetization direction in the solid. The X-ray magnetic circular and linear 
dichroism are given by $\mu_{+}-\mu_{-}$and $\mu_{\|}-\left(\mu_{+}+\mu_{-}\right) / 2$, respectively. Detailed expressions of the matrix elements in the electric dipole approximation may be found in [26-29]. The matrix elements due to the magnetic dipole and electric quadrupole corrections are presented in [29].

Magnetocrystalline anisotropy energy. The internal energy of a ferromagnetic material depends on the direction of spontaneous magnetization. The magnetocrystalline anisotropy energy (MAE), which possesses the crystal symmetry of a material, is a part of this energy. The MAE is an important property that describes the tendency of the magnetization to align along specific spatial directions rather than to randomly fluctuate over time. The MAE determines the stability of magnetization in bulk as well as nanoparticle systems. Extensive studies on ferromagnetic bulk materials and thin films have highlighted the MAE dependence on crystal symmetry and atomic composition. While the exchange interaction among electron spins is purely isotropic, the orbital magnetization connects the spin magnetization to the atomic structure of a magnetic material via the spin-orbit interaction, giving rise to the magnetic anisotropy [30]. The calculation of the magnetocrystalline anisotropy energy has been a long-standing problem. A first theory of the MAE in Fe and Ni was formulated by Brooks [31] and Fletcher [32], who emphasized that the energy band picture in which the effect of spin-orbit (SO) coupling is taken into account in a perturbative way could provide a coupling of the magnetization orientation to the crystallographic axes of approximately the right order of magnitude. In this pioneering work, the band structure was oversimplified to three empirical bands [31, 32]. Recent investigations [33-37] elaborated the MAE problem using $a b$ initio calculated energy bands obtained within the local-spin density approximation to the density functional theory. Although it is beyond doubt that LSDA energy bands are superior to empirical bands, it turned out that the calculation of the MAE from first principles poses a great computational challenge. The prime obstacle is the smallness of the MAE, which is of only a few meV/atom, a value that ought to result from the difference of two total energies for different magnetization directions, which are both of the order of $10^{4} \mathrm{eV} /$ atom. Due to this numerical problem, it remained at first unclear if the LSDA could at all describe the MAE correctly, since the wrong easy axis was obtained for hcp Co and fcc Ni [33]. Recent contributions aimed consequently at improving the numerical techniques [35, 38], with the result that the correct easy axis was obtained for hcp Co, but not for fcc Ni [35]. Halilov et al. [36] reported an ab initio investigation of the magnetocrystalline anisotropy energy in bcc Fe and fcc Co and Ni. They introduced a spin-orbit scaling technique that yielded the correct easy axis for Fe and Co, but a vanishing MAE for Ni. as [39]

For the material exhibiting uniaxial anisotropy, such as a hexagonal crystal, the MAE can be expressed

$$
E(\theta)=K_{1} \sin ^{2} \theta+K_{2} \sin ^{4} \theta+K_{3}^{\prime} \sin ^{6} \theta+K_{3} \sin ^{2} \theta \cos [6(\phi+\psi)]+\cdots,
$$

where $K_{i}$ is the anisotropy constant of the $i$ th order, $\theta$ and $\phi$ are the polar angles of the Cartesian coordinate system where the $c$ axis coincides with the $z$ axis (the Cartesian coordinate system is chosen so that the $x$ axis is rotated through $90^{\circ}$ from the $a$ hexagonal axis) and $\psi$ is a phase angle.

Both the magnetic dipole interaction and the SO coupling give rise to the MAE, the former contributing only to the first-order constant $K_{1}$. Here, we deal with the MAE caused only by the SO interaction. Both the magneto-optical effects and the MAE have a common origin in the SO coupling and exchange splitting. Thus, a close connection between the two phenomena seems plausible.

In this paper, the MAE is defined as the difference between two self-consistently calculated fully relativistic total energies for two different magnetic field directions, $E(\theta)-E_{\langle 001\rangle}$.

Crystal structure. $\quad \mathrm{LaCO}_{2} \mathrm{P}_{2}$ and $\mathrm{PrCO}_{2} \mathrm{P}_{2}$ belong to the $\mathrm{ThCr}_{2} \mathrm{Si}_{2}$ body-centered tetragonal structure type (figure 1). The space group is I4/mmm (No. 139) with $\operatorname{Pr}(\mathrm{La})$ at the $2 a$ positions $(0,0,0)$, Co at the $4 a$ positions $\left(0, \frac{1}{2}, \frac{1}{4}\right)$ and $\mathrm{P}$ at the $4 e$ positions $(0,0, z) ; z=0.3697$ and 0.3568 in $\operatorname{PrCo}_{2} \mathrm{P}_{2}$ and $\mathrm{LaCo}_{2} \mathrm{P}_{2}, \mathrm{re}-$ spectively [16, 17]. In this structure, the transition metal atoms (Co) form planar square nets, and the nonmetal atoms (P) cap the centers of the squares above and below the planes in a chessboard-like fashion. The resulting $\left[\mathrm{CO}_{2} \mathrm{P}_{2}\right]$ layers are separated by layers of rare-earth metal cations ( $\mathrm{Pr}$ or $\mathrm{La}$ ). The $\mathrm{Pr}$ atom in $\mathrm{PrCO}_{2} \mathrm{P}_{2}$ has $8 \mathrm{P}$ nearest neighbors at the distance of $3.0329 \AA$ and 8 Co atoms at the $3.1094 \AA$ distance, the shortest P-P distance is equal to $2.5247 \AA$. In $\mathrm{LaCO}_{2} \mathrm{P}_{2}$, the La atom has $8 \mathrm{P}$ nearest neighbors 


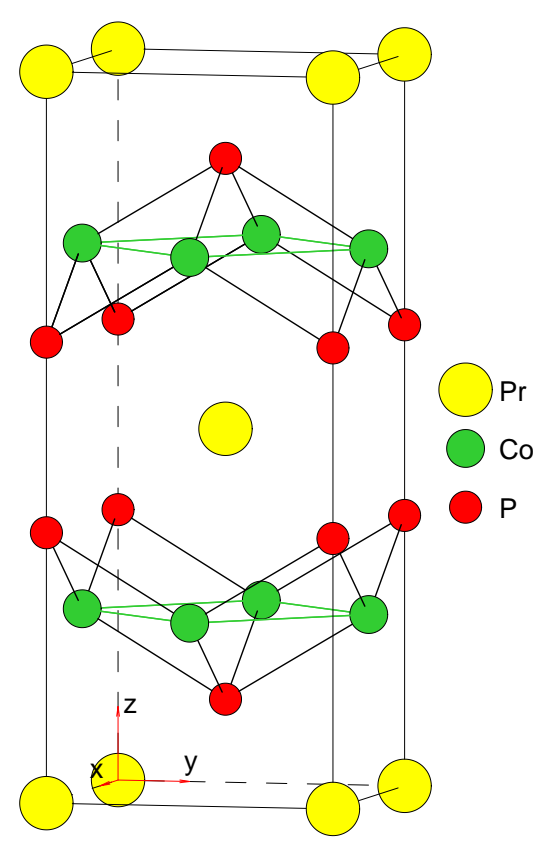

Figure 1. (Color online) Schematic representation of the $\mathrm{PrCO}_{2} \mathrm{P}_{2}$ structure.

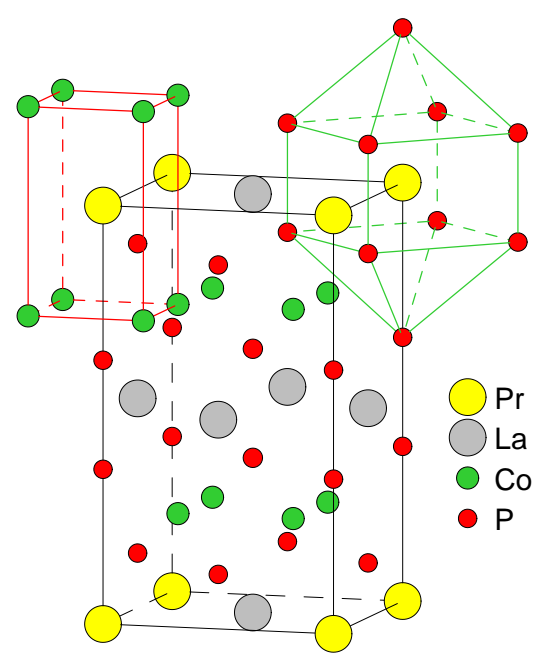

Figure 2. (Color online) Schematic representation of the $\mathrm{La}_{1-x} \mathrm{Pr}_{x} \mathrm{Co}_{2} \mathrm{P}_{2}(x=0.25)$ structure.

at the distance of $3.1265 \AA$ and $8 \mathrm{Co}$ atoms at the $3.3551 \AA$ distance, the P-P distance in $\mathrm{LaCo}_{2} \mathrm{P}_{2}$ is much larger and is equal to $3.1621 \AA$.

The calculations of the energy band structure of $\mathrm{La}_{1-x} \mathrm{Pr}_{x} \mathrm{Co}_{2} \mathrm{P}_{2}(x=0,0.25$, and 1$)$ compounds were performed for $a \times a \times 2 c$ supercells of the tetragonal structure with space group of $\mathrm{P} 4 / \mathrm{mmm}$ (No. 123). The structure refinement parameters of $\mathrm{La}_{1-x} \mathrm{Pr}_{x} \mathrm{Co}_{2} \mathrm{P}_{2}$ compounds are presented in [18]. The crystal structure of $\mathrm{La}_{0.75} \mathrm{Pr}_{0.25} \mathrm{Co}_{2} \mathrm{P}_{2}$ is presented in figure2 2 The $\mathrm{Pr}$ atom in this compound has $8 \mathrm{P}$ nearest neighbors at the distance of $3.0329 \AA$ and $8 \mathrm{Co}$ atoms at the $3.1094 \AA$ distance. The Co atoms are surrounded by $4 \mathrm{P}$ atoms at the distance of $2.2688 \AA$ and 4 Co atoms at the distance of $2.7577 \AA, 4 \mathrm{La}$ atoms are at the $3.1094 \AA$ distance from Co.

Calculation details. The calculations presented in this work were performed using the spin-polarized fully relativistic linear muffin-tin orbital (SPR LMTO) method [40-42] for the experimentally observed lattice constants: $a=3.6 \AA, c=9.688 \AA$ for $\mathrm{PrCo}_{2} \mathrm{P}_{2}$ [16], $a=3.8145 \AA, c=11.041 \AA$ for $\mathrm{LaCo}_{2} \mathrm{P}_{2}$ [17], and $a=3.8260 \AA, c=10.9031 \AA$ for $\mathrm{La}_{1-x} \mathrm{Pr}_{x} \mathrm{Co}_{2} \mathrm{P}_{2}(x=0.25)[24]$. The basis consisted of the $\operatorname{Pr}(\mathrm{La}) s, p, d$, and $f$; Co $s, p$, and $d$; P $s, p$, and $d$ LMTO's. The k-space integrations were performed with the improved tetrahedron method [43], and the self-consistent charge density was obtained with 1063 irreducible k-points. To attain good convergence in total energy, a large number of $\mathbf{k}$ points should be used in calculations. To resolve the difference in total energies and to investigate the magnetocrystalline anisotropy, we used $13824 \mathbf{k}$ points in the irreducible part of the Brillouin zone, which corresponds to 82944 tetrahedra in the full zone.

The X-ray absorption and dichroism spectra were calculated taking into account the exchange splitting of core levels. We also take into account the core-hole effect in the final state using the supercell approximation. The similar approximation has been used by several authors [44, 45]. The finite lifetime of a core hole was accounted for by folding the spectra with a Lorentzian. The widths of core level spectra $\Gamma_{L_{2}}=1.13 \mathrm{eV}$ and $\Gamma_{L_{3}}=0.47 \mathrm{eV}$ for Co, and $\Gamma_{M_{4,5}}=0.75 \mathrm{eV}$ for Pr were taken from [46]. The finite apparative resolution of a spectrometer was accounted for by a Gaussian with the width of $0.6 \mathrm{eV}$.

It is well known that the LSDA fails to describe the electronic structure and properties of the systems in which the interaction among the electrons is strong. In recent years, more advanced methods of electronic structure determination such as the LSDA plus self-interaction corrections (SIC-LSDA) [29, 47], the 
LSDA $+U$ [25] method, the GW approximation [48] and the dynamical mean-field theory (DMFT) 499-51] have sought to remedy this problem and have met considerable success. The LSDA $+U$ method is the simplest among them and most frequently used in the literature. We used the "relativistic" generalization of the LSDA $+U$ method which takes into account the spin-orbit coupling so that the occupation matrix of localized electrons becomes non-diagonal in spin indexes. This method is described in detail in our previous paper [52] including the procedure to calculate the screened Coulomb $U$ and exchange $J$ integrals, as well as the Slater integrals $F^{2}, F^{4}$, and $F^{6}$.

The screened Coulomb $U$ and exchange $J$ integrals enter the LSDA $+U$ energy functional as external parameters and should be determined independently. The value of $U$ can be estimated from the photoemission spectroscopy (PES) and X-ray Bremsstrahlung Isochromat Spectroscopy (BIS) experiments. Because of the difficulties with unambiguous determination of $U$, it can be considered as a parameter of the model. Then, its value can be adjusted to achieve the best agreement of the results of LSDA+ $U$ calculations with PES or optical spectra [53]. While the use of an adjustable parameter is generally considered an anathema among first principles practitioners, the LSDA $+U$ approach does offer a plausible and practical method to treat approximately strongly correlated orbitals in solids. The Hubbard $U$ and exchange parameter $J$ can be determined from supercell LSDA calculations using the Slater's transition state technique [54, 55] or from constrained LSDA calculations [55-57]. Cococcioni and Gironcoli [58] have also provided an internally consistent, basis-set independent method based on the linear response approach for the calculation of the effective interaction parameters in the LSDA $+U$ method. The constrained LSDA calculations produce $U=6.17 \mathrm{eV}, J=0.87 \mathrm{eV}$ for $\operatorname{Pr}$ and $U=4.1 \mathrm{eV}, J=0.81 \mathrm{eV}$ for Co in $\operatorname{PrCo}_{2} \mathrm{P}_{2}$, as well as $U=6.08 \mathrm{eV}, J=0.83 \mathrm{eV}$ for $\mathrm{La}$ and $U=4.0 \mathrm{eV}, J=0.8 \mathrm{eV}$ for Co in $\mathrm{LaCo}_{2} \mathrm{P}_{2}$. These values of $U$ and $J$ were used in our calculations presented below.

\section{Results}

\subsection{Electronic structures of $\mathrm{LaCO}_{2} \mathrm{P}_{2}$ and $\mathrm{PrCO}_{2} \mathrm{P}_{2}$}

Figure 3 presents the partial density of states (PDOS) for $\mathrm{LaCO}_{2} \mathrm{P}_{2}$ and $\mathrm{PrCo}_{2} \mathrm{P}_{2}$. The P $3 s$ states are located mostly between $-13.6 \mathrm{eV}$ and $-10.0 \mathrm{eV}$ below the Fermi level (not shown), while the $3 p$ states of $\mathrm{P}$ are found between $-6.8 \mathrm{eV}$ and $6.1 \mathrm{eV}$ in $\mathrm{LaCo}_{2} \mathrm{P}_{2}$ and between $-7.5 \mathrm{eV}$ and $5.8 \mathrm{eV}$ in $\mathrm{PrCO}_{2} \mathrm{P}_{2}$. The spin splitting of the P $3 p$ states is quite small. The Co $3 d$ states occupy the energy interval between $-6.9 \mathrm{eV}$ and $5.5 \mathrm{eV}$ and hybridize strongly with the P $3 p$ states. The La $4 f$ empty states occupy the $3.2-4.5 \mathrm{eV}$ energy interval in $\mathrm{LaCO}_{2} \mathrm{P}_{2}$. The Pr $4 f$ empty states are from $1.5 \mathrm{eV}$ to $4.8 \mathrm{eV}$ above the Fermi level. Two $\operatorname{Pr} 4 f$ spin-up electrons occupy a small energy interval around $-3 \mathrm{eV}$.

The crystal field at the Co site ( $D_{2 d}$ point symmetry) in both compounds causes the splitting of Co $3 d$ orbitals into three singlets $a_{1}\left(d_{3 z^{2}-1}\right), b_{1}$ $\left(d_{x y}\right), b_{2}\left(d_{x^{2}-y^{2}}\right)$ and a doublet $e\left(d_{y z}\right.$ and $\left.d_{x z}\right)$. A two peak structure of the minority- and majorityspin Co $d$ states is found in the close vicinity of the Fermi energy. We found that the two peaks (occupied in the majority-spin channel at $-0.5 \mathrm{eV}$ and

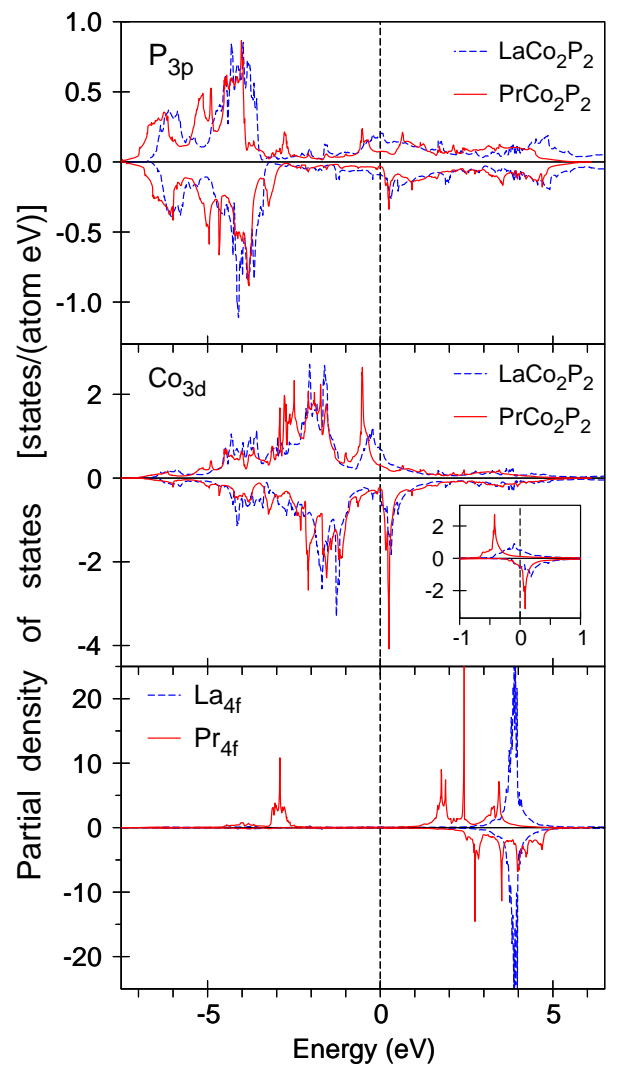

Figure 3. (Color online) Partial density of states of $\mathrm{LaCo}_{2} \mathrm{P}_{2}$ and $\mathrm{PrCo}_{2} \mathrm{P}_{2}$. The insert in the middle panel shows the energy distribution of the $x y$ orbitals. 
empty in the minority-spin channel at $0.3 \mathrm{eV}$ ) are of the $x y$ character. These peaks are rather broad in $\mathrm{LaCO}_{2} \mathrm{P}_{2}$ and very narrow and intensive in $\mathrm{PrCO}_{2} \mathrm{P}_{2}$. Such a difference can be explained by different nearest-neighbors interatomic $\mathrm{Co}-\mathrm{Co}$ and $\mathrm{Co}-\mathrm{P}$ distances. The $\mathrm{Co}-\mathrm{Co}$ distance is equal to $2.697 \AA$ and $2.757 \AA$ in $\mathrm{LaCO}_{2} \mathrm{P}_{2}$ and $\mathrm{PrCO}_{2} \mathrm{P}_{2}$, respectively. Also, the Co-P distance is larger in $\mathrm{LaCo}_{2} \mathrm{P}_{2}$ than in $\mathrm{PrCO}_{2} \mathrm{P}_{2}$ by $0.027 \AA$.

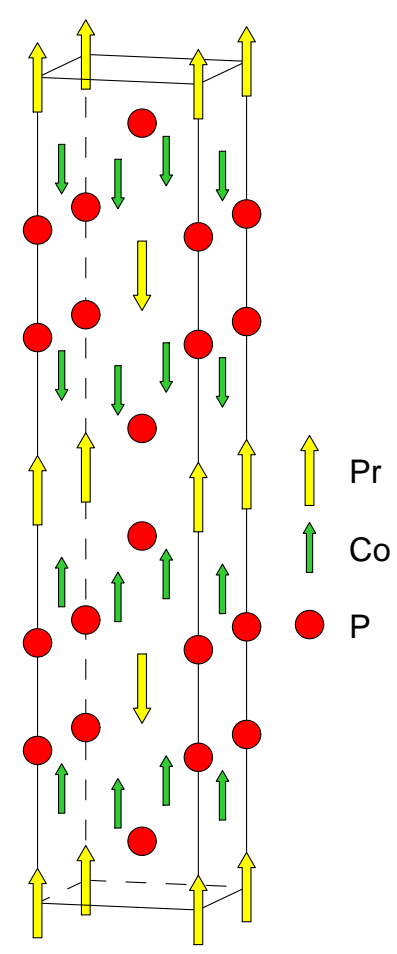

(a)

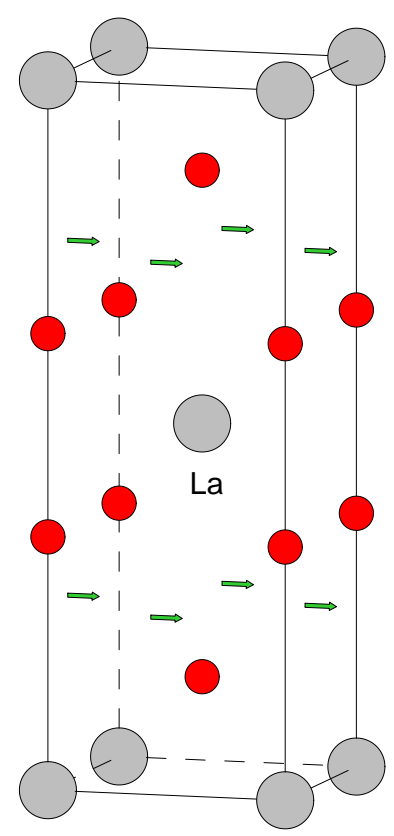

(b)

Figure 4. (Color online) Schematic representation of the magnetic ordering in $\mathrm{PrC}_{2} \mathrm{P}_{2}$ (left-hand column) and $\mathrm{LaCO}_{2} \mathrm{P}_{2}$ (right-hand column).

Our self-consistent calculations reveal a ferromagnetic arrangement of magnetic moments in $\operatorname{PrCo}_{2} \mathrm{P}_{2}$. The spin magnetic moments at the Pr and Co sites are aligned along the $c$ axis with the antiparallel magnetic coupling between the neighboring planes resulting in antiferromagnetism (left-hand column of figure 4. The similar magnetic arrangement was observed experimentally by Reehuis et al. [16]. The theoretical calculations give a ferromagnetic arrangement of magnetic moments in $\mathrm{LaCo}_{2} \mathrm{P}_{2}$, the Co moments are aligned in-plane and parallel to the moments in the other layers (right-hand column of figure 4). Figure 5 (upper panel) shows the MAE as a function of the polar angle $\theta$. The minimum of the total energy corresponds to the magnetic configuration with the Co moments aligned in-plane in agreement with the experimental observation [17]. The theory produces quite a small value of MAE of around $0.28 \mathrm{meV}$ per formula unit in $\mathrm{LaCO}_{2} \mathrm{P}_{2}$. The lower panel of figure 5 shows the anisotropy of spin magnetic moments at the Co site in $\mathrm{LaCO}_{2} \mathrm{P}_{2}$ as a function of the polar angle $\theta$.

The spin $m_{s}$ and orbital $m_{l}$ magnetic moments at the Co site in $\operatorname{PrCo}_{2} \mathrm{P}_{2}$ are larger than in $\mathrm{LaCo}_{2} \mathrm{P}_{2}$. Our band structure calculations yield the magnetic moments for the Co atoms $m_{s}=0.825 \mu_{\mathrm{B}}, m_{l}=0.120$ $\mu_{\mathrm{B}}$ in $\mathrm{PrCo}_{2} \mathrm{P}_{2}$ and $m_{s}=0.665 \mu_{\mathrm{B}}, m_{l}=0.066 \mu_{\mathrm{B}}$ in $\mathrm{LaCo}_{2} \mathrm{P}_{2}$. The induced spin magnetic moments at the $\mathrm{P}$ site are of $0.011 \mu_{\mathrm{B}}$ and $0.016 \mu_{\mathrm{B}}$ for the $\mathrm{PrCo}_{2} \mathrm{P}_{2}$ and $\mathrm{LaCo}_{2} \mathrm{P}_{2}$, respectively. The orbital moments at the $\mathrm{P}$ sites are small in both compounds $\left(m_{l}^{\mathrm{P}}=0.005 \mu_{\mathrm{B}}\right)$. The orbital magnetic moment at the La site is extremely small $m_{l}^{\mathrm{La}}=-0.002 \mu_{\mathrm{B}}$, however, we found quite a large orbital moment at the Pr site in $\mathrm{PrCO}_{2} \mathrm{P}_{2}\left(m_{l}^{\mathrm{Pr}}=0.509 \mu_{\mathrm{B}}\right)$. 


\subsection{Electronic structure, X-ray absorption and XMCD spectra in $\mathrm{La}_{1-x} \mathrm{Pr}_{x} \mathrm{CO}_{2} \mathrm{P}_{2}$ com- pounds}

Figure 6 presents the partial density of states in $\mathrm{La}_{1-x} \mathrm{Pr}_{x} \mathrm{Co}_{2} \mathrm{P}_{2}$ for $x=0.25$. The energy position and shape of PDOS slightly differ from the corresponding PDOS of $\mathrm{LaCo}_{2} \mathrm{P}_{2}$ and $\mathrm{PrCo}_{2} \mathrm{P}_{2}$. The P $3 p$ PDOS has more pronounced peaks near the Fermi level due to the larger P $3 p-$ Co $3 d$ hybridization. The positions of the empty La and $\operatorname{Pr} 4 f$ states are shifted closer to the Fermi level in comparison with the reference compounds. Also, the shape of the energy distribution of $\operatorname{Pr} 4 f$ states slightly differ from the corresponding $4 f$ PDOS in $\mathrm{PrCo}_{2} \mathrm{P}_{2}$ and $\mathrm{LaCO}_{2} \mathrm{P}_{2}$. Such differences might be explained by different lattice constants and different numbers and distances of the nearest neighbors.

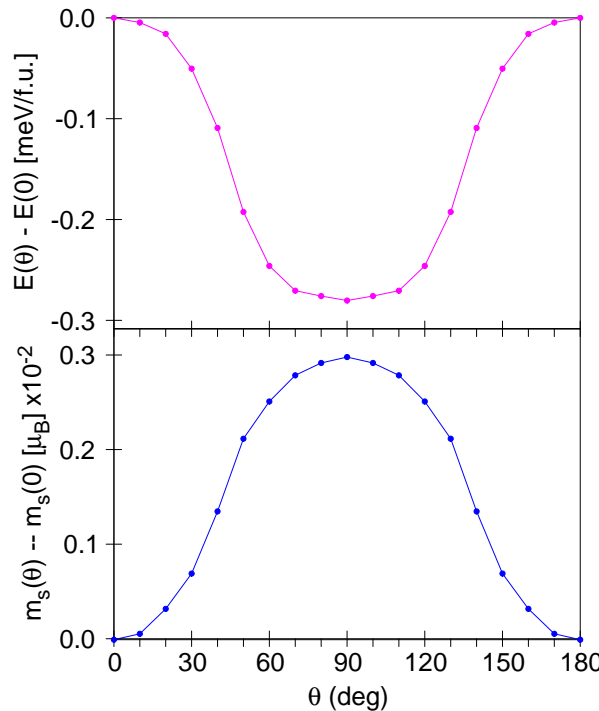

Figure 5. (Color online) MAE (upper panel) and anisotropy of spin magnetic moments at the Co site (lower panel) in $\mathrm{LaCO}_{2} \mathrm{P}_{2}$ as functions of the polar angle $\theta$.

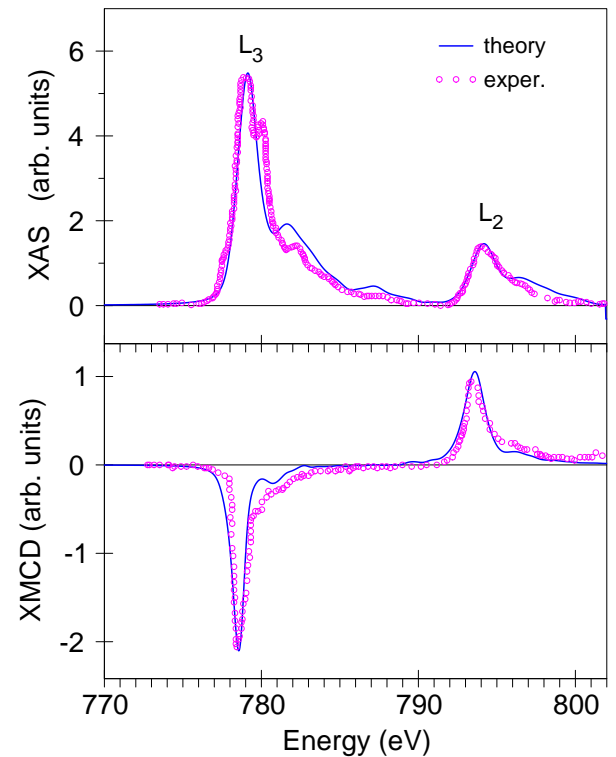

Figure 6. (Color online) Partial density of states in $\mathrm{La}_{1-x} \mathrm{Pr}_{x} \mathrm{Co}_{2} \mathrm{P}_{2}$ for $x=0.25$.

The spin magnetic moments at the Pr and Co sites (1.955 $\mu_{\mathrm{B}}$ and $0.815 \mu_{\mathrm{B}}$, respectively) are aligned along the $c$ axis in $\mathrm{La}_{0.75} \mathrm{Pr}_{0.25} \mathrm{Co}_{2} \mathrm{P}_{2}$ with antiparallel magnetic moments at the La sites $\left(m_{s}=-0.002 \mu_{\mathrm{B}}\right.$ and $-0.007 \mu_{\mathrm{B}}$ for two nonequivalent La sites). The orbital magnetic moments are equal to $0.054 \mu_{\mathrm{B}}$, $0.109 \mu_{\mathrm{B}}$, and $0.020 \mu_{\mathrm{B}}$ for Pr, Co, and La sites, respectively. The orbital moments at the P sites are small $\left(m_{l}^{\mathrm{P}}=0.001 \mu_{\mathrm{B}}\right)$.

Figure 7 (upper panel) shows the X-ray absorption spectra at the Co $L_{2,3}$ edges in $\operatorname{La}_{0.75} \operatorname{Pr}_{0.25} \mathrm{Co}_{2} \mathrm{P}_{2}$

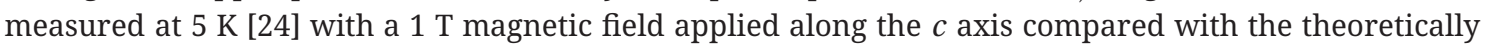
calculated ones in the LSDA $+U$ approximation. The Co $L_{3} \mathrm{X}$-ray absorption spectrum possesses four major fine structures: a major peak at $779 \mathrm{eV}$ and three high energy shoulders at $780.5 \mathrm{eV}, 782.5 \mathrm{eV}$ and $787.5 \mathrm{eV}$. The theory describes reasonably well the energy position and relative intensity of all the fine structures except the shoulder at $780.5 \mathrm{eV}$. Due to the electric dipole selection rules $(\Delta l= \pm 1 ; \Delta j=0, \pm 1)$, the major contribution to the absorption at the $L_{3}$ edge stems from the transitions $2 p_{3 / 2} \rightarrow 5 d_{5 / 2}$, with a weaker contribution from the $2 p_{3 / 2} \rightarrow 5 d_{3 / 2}$ transitions. For the latter case, the corresponding $2 p_{3 / 2} \rightarrow$ $5 d_{3 / 2}$ radial matrix elements are only slightly smaller than for the $2 p_{3 / 2} \rightarrow 5 d_{5 / 2}$ transitions. The angular matrix elements, however, strongly suppress the $2 p_{3 / 2} \rightarrow 5 d_{3 / 2}$ contribution. Therefore, the contribution to the XAS spectrum at the $L_{3}$ edge from the transitions with $\Delta j=0$ is one order of magnitude smaller than the transitions with $\Delta j=1$ [28]. 
The lower panel of figure 7 presents the XMCD experimental spectra of $\operatorname{La}_{0.75} \operatorname{Pr}_{0.25} \mathrm{Co}_{2} \mathrm{P}_{2}$ at the Co $L_{2,3}$ edges and the theoretically calculated ones. The LSDA $+U$ calculations describe reasonably well all the features of the experimental XMCD spectra.

A study of the $4 f$ electron shell in rare earth compounds is usually performed by tuning the energy of X-ray close to the $M_{4,5}$ edges of rare-earth where the electronic transitions between $3 d_{3 / 2,5 / 2}$ and $4 f_{5 / 2,7 / 2}$ states are involved. Figure 8 shows the XAS and XMCD spectra at the $\operatorname{Pr} M_{4,5}$ edges in $\operatorname{La}_{0.75} \operatorname{Pr}_{0.25} \mathrm{Co}_{2} \mathrm{P}_{2}$ measured at $5 \mathrm{~K} \mathrm{[24]}$ with a $1 \mathrm{~T}$ magnetic field applied along the $c$ axis compared with the theoretically calculated ones in the LSDA $+U$ approximation. The Dirac-Hartree-Fock-Slater single-particle approximation used in this work to calculate the core states is not capable of producing a correct energy position of the spectra (because the self-interaction correction, different kinds of relaxation processes and other many-particle effects were not taken into account). Therefore, we used the experimentally measured positions of the spectra. The theoretically calculated XAS spectra have a rather simple line shape composed of two white line peaks at the $M_{5}$ and $M_{4}$ edges. The theory reproduces the shape of the $M_{5}$ XAS spectrum very well. However, the experimentally measured $M_{4}$ spectrum has a well pronounced fine structure at the low energy part that is not reproduced by the theory. We should mention here that a major shortcoming in the band structure approximation is that the multiplet structure is not included. For the Co $L_{2,3}$ edges, this is not the major problem. However, for the $\operatorname{Pr} M_{4,5}$ edges, the core-valence electrostatic interactions can significantly effect the line shape of the XAS and XMCD spectra. The fine structure on the low energy side of $\operatorname{Pr} M_{4}$ XASs is believed to be due to the multiplet structure, which is not included in our calculations. A theoretical method that consistently includes both the band structure and the atomic-like multiplet structure of rare earth metals and compounds is highly desired. However, we should mention that it is still not clear why the multiplet structure is pronounced only at the $\operatorname{Pr} M_{4}$ edge and is not seen at the $M_{5}$ edge.

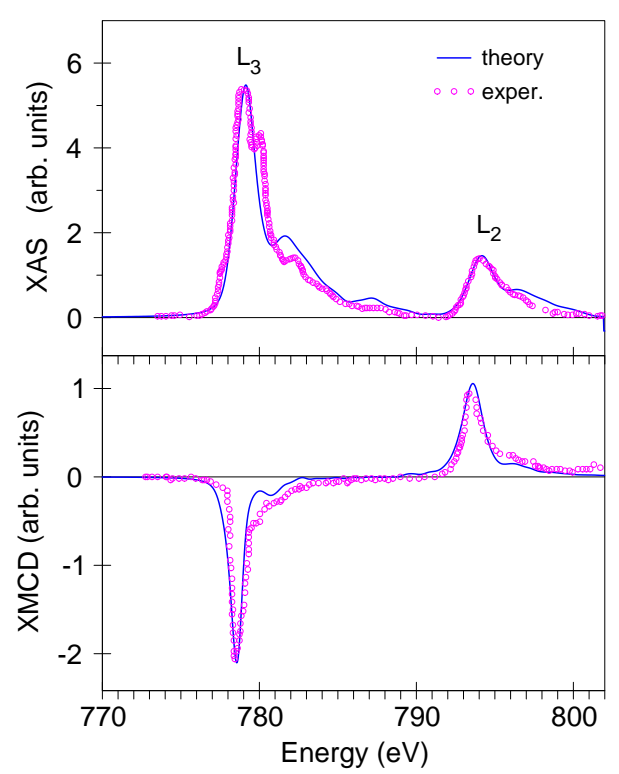

Figure 7. (Color online) Experimental and theoretically calculated X-ray absorption (upper panel) and XMCD (lower panel) spectra of $\mathrm{La}_{0.75} \operatorname{Pr}_{0.25} \mathrm{Co}_{2} \mathrm{P}_{2}$ at the $\mathrm{Co}_{2,3}$ edges.

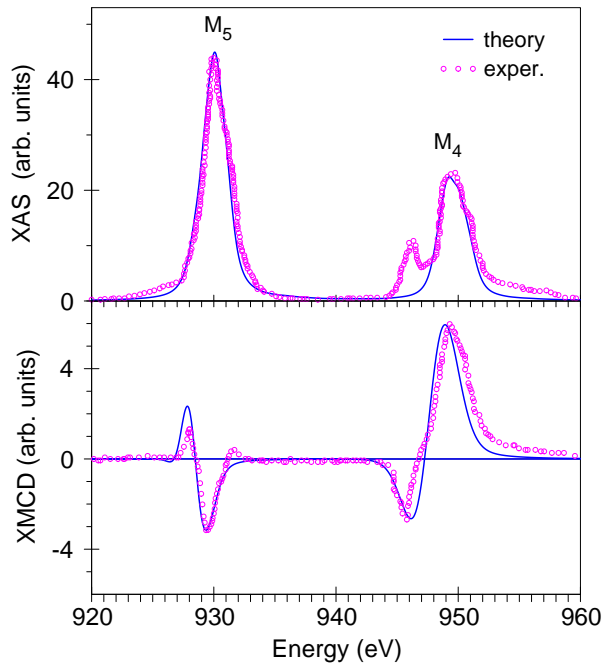

Figure 8. (Color online) Experimental and theoretically calculated X-ray absorption (upper panel) and XMCD (lower panel) spectra of $\mathrm{La}_{0.75} \mathrm{Pr}_{0.25} \mathrm{Co}_{2} \mathrm{P}_{2}$ at the $\operatorname{Pr} M_{4,5}$ edges.

Figure 8 (lower panel) shows the calculated Pr $M_{4,5}$ XMCD spectra in the LSDA $U$ approximation for $\mathrm{La}_{0.75} \mathrm{Pr}_{0.25} \mathrm{CO}_{2} \mathrm{P}_{2}$ together with the corresponding experimental data [24]. The experimentally measured dichroism is rather large. The XMCD spectra at the $M_{5}$ and $M_{4}$ edges have a two-peak structure. The dichroism is positive at a lower energy and negative at a higher energy at the $\operatorname{Pr} M_{5}$ edge, while the $\operatorname{Pr} M_{4}$ XMCD spectrum has a negative minimum at a lower energy and a positive maximum at a higher 
energy. The LSDA $+U$ calculations quite well describe all the features of the experimental XMCD spectra at the $M_{4,5}$ edges. The theory also correctly reproduces the relative intensities of the XAS and XMCD spectra at the $M_{5}$ and $M_{4}$ edges, namely, the XAS is larger at the $M_{5}$ edge than at the $M_{4}$ edge, while the XMCD spectra show the opposite behavior with larger dichroism at the $M_{4}$ edge in comparison with the $M_{5}$ edge.

When a $3 d$ core-electron is photo-excited to an unoccupied $4 f$ state, the distribution of the charge changes to account for the created hole. We investigated this core-hole effect in the final state using the supercell approximation. We found that the final-state interaction has little effect on the shape of the XAS and XMCD spectra at the $\operatorname{Pr} M_{4,5}$ edges. We also investigated the effect of the electric quadrupole $E_{2}$ and magnetic dipole $M_{1}$ transitions. We found that the $M_{1}$ transitions are extremely small in comparison with the $E_{2}$ transitions and can be neglected. The $E_{2}$ transitions are much weaker than the electric dipole transitions $E_{1}$. They are almost invisible in the XAS and have a very small effect on the XMCD spectra at the $\operatorname{Pr} M_{4,5}$ edges.

\section{Summary}

The electronic structure and magnetic ordering in $\mathrm{La}_{1-x} \mathrm{Pr}_{x} \mathrm{Co}_{2} \mathrm{P}_{2}(x=0,0.25$, and 1$)$ phosphides have been studied theoretically using the fully relativistic spin-polarized Dirac LMTO band-structure method. The self-consistent calculations reveal a ferromagnetic arrangement of magnetic moments in $\operatorname{PrCO}_{2} \mathrm{P}_{2}$. The spin magnetic moments at the Pr and Co sites are aligned along the $c$ axis with the antiparallel magnetic coupling between the neighboring planes resulting in antiferromagnetism. $\mathrm{LaCo}_{2} \mathrm{P}_{2}$ possesses a ferromagnetic arrangement of magnetic moments where the Co moments are aligned in-plane and parallel to the moments in the other layers. The theory gives quite a small value of $\mathrm{MAE}$ in $\mathrm{LaCO}_{2} \mathrm{P}_{2}$ (around $0.28 \mathrm{meV}$ per formula unit).

We have studied the X-ray magnetic circular dichroism at the Co $L_{2,3}$ and $\operatorname{Pr} M_{4,5}$ edges in $\mathrm{La}_{0.75} \mathrm{Pr}_{0.25} \mathrm{Co}_{2} \mathrm{P}_{2}$. The calculations show good agreement with the experimental measurements. The core-hole effect was found to be very small on the shape of the XAS and XMCD spectra at the Pr $M_{4,5}$ edges. We found that the magnetic dipole $M_{1}$ transitions are extremely small in comparison with the electric quadrupole $E_{2}$ transitions and can be neglected. The $E_{2}$ transitions were found to be weaker than the electric dipole transitions $E_{1}$. They are almost invisible in the XAS and have a very small effect on the XMCD spectra at the $\operatorname{Pr} M_{4,5}$ edges.

\section{References}

1. Villars P., Calvert L.D., Pearson’s Handbook of Crystallographic Data for Intermetallic Phases, ASM International, Metals Park, Ohio, 1985.

2. Custers J., Gegenwart P., Wilhelm H., Neumaier K., Tokiwa Y., Trovarelli O., Geibel C., Steglich F., Pépin C., Coleman P., Nature, 2003, 424, 524; doi 10.1038/nature01774

3. Ren Z.-A., Lu W., Yang J., Yi W., Shen X.-L., Zheng G.-C.C., Dong X.-L., Sun L.-L., Zhou F., Zhao Z.-X., Chinese Phys. Lett., 2008, 25, 2215; doi 10.1088/0256-307X/25/6/080.

4. Rotter M., Tegel M., Johrendt D., Phys. Rev. Lett., 2008, 101, 107006; doi 10.1103/PhysRevLett.101.107006

5. Szytula A., Leciejewicz J., In: Handbook of the Physics and Chemistry of Rare Earths, Gschneidner K. A., Eyring L. (Eds.), 12, North-Holland, Amsterdam, 1989, 133.

6. Parthe E., Chabot B., In: Handbook of the Physics and Chemistry of Rare Earths, Gschneidner K. A., Eyring L. (Eds.), 6, North-Holland, Amsterdam, 1984, 113.

7. Fujii H., Okamoto T., Shigeoka T., Iwata N., Solid State Commun., 1985, 53, 715; doi 10.1016/0038-1098(85)90385-0.

8. Duraj M., Duraj R., Szytula A. J., J. Magn. Magn. Mater., 1989, 79, 61; doi 10.1016/0304-8853(89)90292-8

9. Morellon L., Algarabel P.A., Ibarra M.R., Ritter C., Phys. Rev. B, 1997, 55, 12363; doi 10.1103/PhysRevB.55.12363

10. Wang Y.G., Yang F., Chen C., Tang N., Wang Q., J. Phys.: Condens. Matter, 1997, 9, 8539; doi $10.1088 / 0953-8984 / 9 / 40 / 019$

11. Duman E., Acet M., Dincer I., Elmali A., Elerman Y., J. Magn. Magn. Mater., 2007, 309, 40; doi $10.1016 /$ j.jmmm.2006.06.010

12. Reehuis M., Jeitschko W., Möller M.H., Brown P., J. Phys. Chem. Solids, 1992, 53, 687; doi 10.1016/0022-3697(92)90208-U 
13. Jeitschko W., Reehuis M., J. Phys. Chem. Solids, 1987, 48, 667; doi 10.1016/0022-3697(87)90157-0

14. Mörsen E., Mosel B. D., Miiller-Warmuth W., Reehuis M., Jeitschko W., J. Phys. Chem. Solids, 1988, 49, 785; doi 10.1016/0022-3697(88)90030-3

15. Reehuis M., Jeitschko W., J. Phys. Chem. Solids, 1990, 51, 961; doi 10.1016/0022-3697(90)90039-I

16. Reehuis M., Brown P.J., Jeitschko W., Möller M.H., Vomhof T., J. Phys. Chem. Solids, 1993, 54, 469; doi 10.1016/0022-3697(93)90330-T

17. Reehuis M., Ritter C., Ballou R., Jeitschko W., J. Magn. Magn. Mater., 1994, 138, 85; doi 10.1016/0304-8853(94)90402-2

18. Kovnir K., Thompson C.M., Zhou H.D., Wiebe C.R., Shatruk M., Chem. Mater., 2010, 22, 1704; doi $10.1021 / \mathrm{cm} 903497 \mathrm{~h}$

19. Kovnir K., Reiff W.M., Menushenkov A.P., Yaroslavtsev A.A., Chernikov R.V., Shatruk M., Chem. Mater., 2011, 23, 3021; doi $10.1021 / \mathrm{cm} 200782 \mathrm{z}$

20. Kovnir K., Garlea V.O., Thompson C.M., Zhou H.D., Reiff W.M., Ozarowski A., Shatruk M., Inorg. Chem., 2011, 50, 10274; doi 10.1021/ic201328y

21. Jia S., Chi S., Lynn J.W., Cava R.J., Phys. Rev. B, 2010, 81, 214446; doi 10.1103/PhysRevB.81.214446

22. Jia S., Jiramongkolchai P., Suchomel M.R., Toby B.H., Checkelsky J.G., Ong N.P., Cava R.J., Nat. Phys., 2011, 7, 207; doi $10.1038 /$ nphys1868

23. Jeitschko W., Meisen U., Möller M.H., Reehuis M.Z., Anorg. Allg. Chem., 1985, 527, 73; doi 10.1002/zaac.19855270807

24. Kovnir K., Thompson C.M., Garlea V.O., Haskel D., Polyanskii A.A., Zhou H., Shatruk M., Phys. Rev. B, 2013, 88, 104429; doi 10.1103/PhysRevB.88.104429

25. Anisimov V.I., Zaanen J., Andersen O.K., Phys. Rev. B, 1991, 44, 943; doi 10.1103/PhysRevB.44.943

26. Guo G.Y., Ebert H., Temmerman W.M., Durham P.J., Phys. Rev. B, 1994, 50, 3861; doi 10.1103/PhysRevB.50.3861

27. Antonov V.N., Bagljuk A.I., Perlov A.Y., Nemoshkalenko V.V., Antonov V.N., Andersen O.K., Jepsen O., Fizika Nizkikh Temperatur, 1993, 19, 689 (in Russian).

28. Antonov V., Harmon B., Yaresko A., Electronic Structure and Magneto-Optical Properties of Solids, Kluwer, Dordrecht, 2004.

29. Arola E., Horne M., Strange P., Winter H., Szotek Z., Temmerman W.M., Phys. Rev. B, 2004, 70, 235127; doi 10.1103/PhysRevB.70.235127

30. Hirjibehedin C., Lin C., Otte A., Ternes M., Lutz C., Jones B., Science, 2007, 317, 1199; doi 10.1126/science.1146110

31. Brooks H., Phys. Rev., 1940, 58, 909; doi 10.1103/PhysRev.58.909

32. Fletcher G.C., Proc. Phys. Soc. A, 1954, 67, 505; doi 10.1088/0370-1298/67/6/303

33. Daalderop G. H.O., Kelly P.J., Schuurmans M.F.H., Phys. Rev. B, 1990, 41, 11919; doi 10.1103/PhysRevB.41.11919

34. Strange P., Staunton J.B., Györffy B.L., Ebert H., Physica B, 1991, 172, 51; doi 10.1016/0921-4526(91)90416-C.

35. Trygg J., Johansson B., Eriksson O., Wills J.M., Phys. Rev. Lett., 1995, 75, 2871; doi 10.1103/PhysRevLett.75.2871.

36. Halilov S.V., Perlov A.Y., Oppeneer P.M., Yaresko A.N., Antonov V.N., Phys. Rev. B, 1998, 57, 9557; doi 10.1103/PhysRevB.57.9557

37. Ravindran P., Delin A., James P., Johansson B., Wills J.M., Ahuja R., Eriksson O., Phys. Rev. B, 1999, 59, 15680; doi $10.1103 /$ PhysRevB.59.15680

38. Wang D.-S., Wu R., Freeman A.J., Phys. Rev. Lett., 1993, 70, 869; doi 10.1103/PhysRevLett.70.869

39. Smit J., Wijn H. P.J., Ferrites, Philips Technical Library, Eindhoven, 1959.

40. Andersen O.K., Phys. Rev. B, 1975, 12, 3060; doi 10.1103/PhysRevB.12.3060

41. Nemoshkalenko V.V., Krasovskii A.E., Antonov V.N., Antonov V.N., Fleck U., Wonn H., Ziesche P., Phys. Status Solidi B, 1983, 120, 283; doi 10.1002/pssb.2221200130

42. Antonov V.N., Perlov A.Y., Shpak A.P., Yaresko A.N., J. Magn. Magn. Mater., 1995, 146, 205; doi $10.1016 / 0304-8853(95) 01083-1$

43. Blöchl P.E., Jepsen O., Andersen O.K., Phys. Rev. B, 1994, 49, 16223; doi 10.1103/PhysRevB.49.16223

44. Brouder C., Alouani M., Bennemann K.H., Phys. Rev. B, 1996, 54, 7334; doi 10.1103/PhysRevB.54.7334

45. Schwitalla J., Ebert H., Phys. Rev. Lett., 1998, 80, 4586; doi 10.1103/PhysRevLett.80.4586

46. Campbell J.L., Parr T., Atom. Data Nucl. Data, 2001, 77, 1; doi 10.1006/adnd.2000.0848

47. Perdew J.P., Zunger A., Phys. Rev. B, 1981, 23, 5048; doi 10.1103/PhysRevB.23.5048

48. Hedin L., Phys. Rev., 1965, 139, A796; doi 10.1103/PhysRev.139.A796

49. Metzner W., Vollhardt D., Phys. Rev. Lett., 1989, 62, 324; doi 10.1103/PhysRevLett.62.324

50. Pruschke T., Jarell M., Freericks J.K., Adv. Phys., 1995, 44, 187; doi 10.1080/00018739500101526

51. Georges A., Kotliar G., Krauth W., Rozenberg M.J., Rev. Mod. Phys., 1996, 68, 13; doi 10.1103/RevModPhys.68.13

52. Yaresko A.N., Antonov V.N., Fulde P., Phys. Rev. B, 2003, 67, 155103; doi 10.1103/PhysRevB.67.155103

53. Bengone O., Alouani M., Blöchl P., Hugel J., Phys. Rev. B, 2000, 62, 16392; doi 10.1103/PhysRevB.62.16392

54. Anisimov V.I., Gunnarsson O., Phys. Rev. B, 1991, 43, 7570; doi 10.1103/PhysRevB.43.7570 
55. Solovyev I.V., Dederichs P.H., Anisimov V.I., Phys. Rev. B, 1994, 50, 16861; doi 10.1103/PhysRevB.50.16861

56. Dederichs P.H., Blügel S., Zeller R., Akai H., Phys. Rev. Lett., 1984, 53, 2512; doi 10.1103/PhysRevLett.53.2512

57. Pickett W.E., Erwin S.C., Ethridge E.C., Phys. Rev. B, 1998, 58, 1201; doi:

58. Cococcioni M., de Gironcoli S., Phys. Rev. B, 2005, 71, 035105; doi 10.1103/PhysRevB.71.035105

\title{
Електронна структура, магнітне впорядкування та рентгенівський магнітний циркулярний дихроїзм у фосфідах $\operatorname{La}_{1-x} \mathrm{Pr}_{x} \mathrm{Co}_{2} \mathrm{P}_{2}$
}

\author{
Л.В. Бекеньов, С.В. Мокляк, В.М. Антонов \\ Інститут металофізики ім. Г.В.Курдюмова НАН України, бульв. Вернадського 36, 03142, Київ \\ На основі зонних розрахунків повністю релятивістським спін-поляризованим лінійним методом МТ- \\ орбіталей (лмТо) теоретично вивчені електронна структура і магнітне впорядкування у фосфідах \\ $\mathrm{La}_{1-x} \mathrm{Pr}_{x} \mathrm{Co}_{2} \mathrm{P}_{2}$ ( $x=0,0.25$, та 1). В рамках методу LSDA+U теоретично досліджені рентгенівські спектри \\ поглинання та спектри рентгенівського циркулярного дихроїзму на краях поглинання $\mathrm{Co} L_{2,3}$ i $\operatorname{Pr} M_{4,5}$. \\ Вивчено вплив остовної дірки в кінцевому стані, а також електричних квадрупольних $E_{2}$ і магнітних ди- \\ польних $M_{1}$ переходів. Отримано добре узгодження з експериментальними результатами.
}

Ключові слова: сильно скорельовані системи, зонна структура, магнітні моменти, рентгенівський магнітний циркулярний дихроїзм 


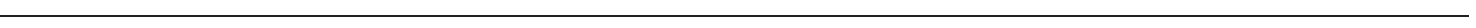

